\title{
A 'Mirage' of a Dislocated Lens in Uveitis
}

\author{
Sana Nadeem ${ }^{1}$, B. A. Naeem ${ }^{2}$ \\ ${ }^{1-2}$ Department of Ophthalmology, Foundation University Medical College/Fauji Foundation Hospital, Rawalpindi
}

\begin{abstract}
We present a very strange case of a 44-year-old diabetic lady who presented with what appeared to be a cataractous lens dislocated in the anterior chamber of her left eye, following weight bearing, a week prior to presentation. The possibility of this being the lens became doubtful on slit lamp examination, as keratic precipitates and flare was noted bilaterally. Her pupil was miosed rendering posterior segment view extremely difficult. Subconjunctival mydriacaine and dexamethasone were given along with topical steroids and cycloplegics, which allowed a better view of the pupillary area, revealing a hint of a nuclear sclerotic cataract, very well in its natural anatomical location. This is a rare presentation of exudate in anterior uveitis presenting as a lens-like mass, producing such an illusion, as to completely baffle doctors regarding its diagnosis. The mass-like exudate subsequently diminished with topical therapy, and disappeared in around a week's time. Both eyes are quiet at a five months' follow-up.
\end{abstract}

Key Words: Anterior Uveitis, Dislocated Lens, Exudate, Diabetes.

How to Cite this Article: Nadeem S, Naeem BA. A 'Mirage' of a Dislocated Lens in Uveitis, Pak J Ophthalmol. 2020; 36 (1): 83-86.

DOI: https://doi.org/10.36351/pjo.v36i1.883.

\section{INTRODUCTION}

Uveitis is a broad term encompassing any inflammation of the uveal tract; iris, ciliary body and choroid, and their surrounding structures. It is the fourth leading cause of blindness ${ }^{1}$ in the developed world, accounting for $10-15 \%$ of preventable blindness ${ }^{2}$ in the West. Its causes are manifold ranging from infections, autoimmune diseases, vasculitis, neoplasia, and drugs; to idiopathic cases, which are the commonest. Anterior uveitis is the most common, amounting to $28-66 \%$ of the cases referred to tertiary hospitals $^{3}$. Idiopathic anterior uveitis has been described several times in poorly controlled diabetic patients, but the association between the two is still questionable ${ }^{4}$. Clinical features of diabetic anterior uveitis ${ }^{5}$ include posterior synechiae, fibrin in anterior

Correspondence to: Sana Nadeem

Assistant Professor, Department of Ophthalmology

Foundation University Medical College/Fauji Foundation

Hospital, Rawalpindi

Email: sana.nadeem@fui.edu.pk chamber, keratic precipitates, folds in Descemet membrane, or hypopyon. We present an unusual case of anterior uveitis in a poorly controlled diabetic lady, who presented with a mirage of a dislocated lens in her anterior chamber, which was in fact exudate.

\section{CASE PRESENTATION}

A 44-year-old lady presented to the OPD of Fauji Foundation Hospital, Rawalpindi; which is a teaching hospital affiliated with the Foundation University Medical College; with sudden pain and loss of vision of the left eye for the past seven days, which occurred when she lifted a heavy object. Examination revealed her visual acuity in the left eye to be hand movements only and 6/36 in the right eye, with the Snellen chart. The anterior chamber seemed to have a moderately cataractous dislocated lens in it, with mild corneal edema. She was admitted immediately for emergency evacuation of the lens. The intraocular pressures were $17 \mathrm{~mm} \mathrm{Hg}$ OD and $10 \mathrm{~mm} \mathrm{Hg}$ OS.

In the ward however, the possibility was raised that this disciform structure might be something else, 


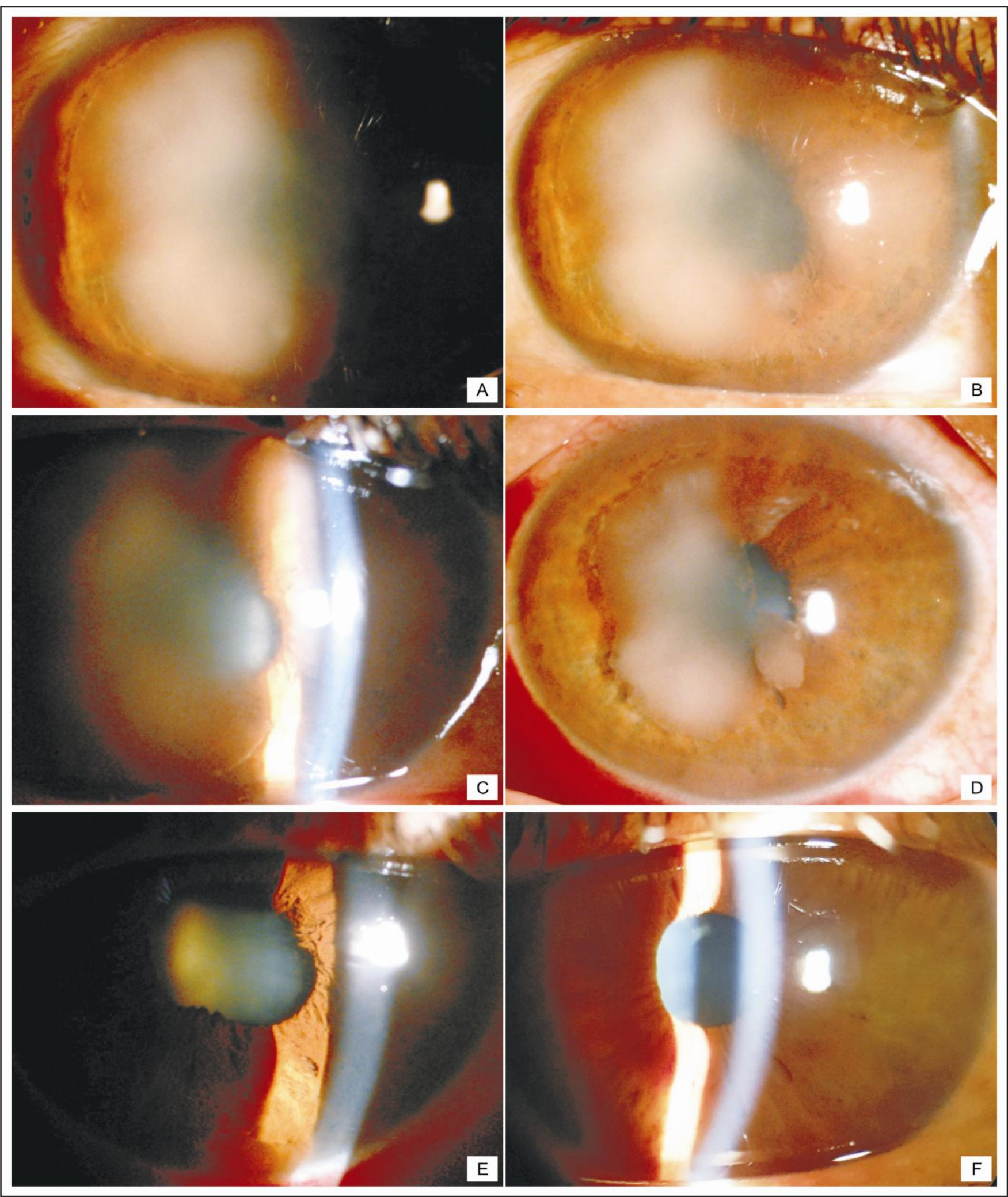

Fig. 1A: Solid lens-like mass in the anterior chamber with well-defined margins touching the cornea. B: The cloudiness of the lenticular mass and margins contributing to the lens-like effect. C: Dilating the pupil with Mydriacaine sub-conjunctival injection, did indeed reveal a nuclear sclerotic natural lens, very much in its natural place. D: Partially resolved exudate after repeated sub-conjunctival steroid injections and topical steroids and cycloplegics on Day 2. E: At Day 4, the exudate has diminished and settled inferiorly. F: Complete resolution of the uveitis at five months follow-up. 
as it was observed, that it was thinner anteroposteriorly than the natural lens, and that the anterior chamber details were also hazy, with +3 cells a few fibrin strands, and few white keratic precipitates inferiorly. The edges of the presumed lens were well defined, except for an indentation inferotemporally, which could be considered a lenticular coloboma, with fibrin filaments attached at the margins (Figure 1). The pupil was miosed and it was not possible to assess the posterior chamber or segment at the slit lamp. The other eye had scant posterior synechiae and +1 cells. The patient was a known diabetic on regular treatment and did not give any prior history of uveitis or its symptoms. It was decided to dilate the pupil with subconjunctival Mydriacaine (lignocaine, adrenaline, and atropine) $0.5 \mathrm{ml}$ injection along with dexamethasone injection (Decadron $\left.{ }^{\circledR}\right) 1 \mathrm{ml}$, to assess the presence of lens in its position and to confirm the possibility of this lens-like mass to be exudate, rather than ectopia lentis. She was also started on half-hourly topical steroid (Prednisolone acetate 1\%) and atropine drops $1 \%$ thrice a day in the left eye, and four times in the right eye. B-scan ultrasound revealed no abnormality of the posterior segment. Half hour later, the pupil only dilated a little due to posterior synechiae, however, the patient's natural crystalline lens which also had nuclear sclerosis, became visible clearly enough to refute the diagnosis of ectopia lentis. Thus, our suspicion was confirmed that this was pseudo ectopia lentis due to intense exudate in the anterior chamber, which had for some reason organized. An enquiry about review of systems for uveitis was negative except for Type 2 diabetes mellitus. The patient was counseled, and routine investigations were undertaken for uveitis; Complete blood count, ESR, Chest X-ray, Mantoux test, and Serum Angiotensin Converting Enzyme (ACE). The exudate decreased on the next day. Sub-conjunctival dexamethasone $1 \mathrm{ml}$ was repeated, and the mass sank down by Day 4 of topical therapy. She had bilateral grade 2 brunescent nuclear sclerosis and mild posterior subcapsular cataract. Fundus examination revealed bilateral mild non-proliferative diabetic retinopathy (NPDR) with clinically significant macular edema (CSME) in the left eye. Her diabetes mellitus was uncontrolled at the time and a medical consultation was sought to normalize here blood sugar levels. She was discharged after resolution of her exudate, on topical steroid four times a day and ketorolac eye drops thrice a day and cyclopentolate eye drops $1 \%$ thrice a day. The drops were tapered soon as her uveitis settled. Her CSME was managed by an intravitreal injection of Bevacizumab $1.25 \mathrm{mg}$ in 0.05 $\mathrm{ml}$ (Avastin). Her investigations for uveitis were negative. Five months later, she had bilaterally quiet anterior chambers. Her best corrected visual acuity was $6 / 60$ OD and 6/18 OS, her posterior subcapsular cataracts and nuclear sclerosis had progressed and cataract surgery was planned in due time. The retinopathy was stable at the moment.

\section{DISCUSSION}

Uveitis has long been described in patients with diabetes mellitus. Little is known about its pathogenesis however in such patients, whether it represents a true inflammation or ischemia ${ }^{6}$ is a causative factor. Elevated intraocular fibronectin ${ }^{7}$ may play a role in such cases. However, poor diabetic control and idiopathic uveitis has been linked ${ }^{8,9}$ with severe anterior segment inflammation and poor vision at presentation, along with diabetic retinopathy; all three features were present in our patient. Bilateral uveitis $^{9}$ is also more common in such patients with a higher complication rate. Dense fibrin ${ }^{9}$ is also reported to be more common in diabetic eyes, as is the case with our patient whose exudate took a lenticular shape, with fibrin strands visible at the margins of the mass.

However, no case of an organized mass in a diabetic patient or uveitis patient has ever been reported in literature. The history of heavy weight bearing added suspicion to delude doctors enough to warrant admission and planning of emergency cataract surgery. Whether the patient's diabetes added to the organization of her anterior chamber reaction, is not known to us, but the question does arise at this time.

Investigations routinely done for uveitis cases, in our set up were all negative, thus raising the possibility of either diabetes as causation, or it could be an idiopathic condition. The patient was fortunate that she was diagnosed correctly and steroid therapy was ensued promptly. The sub-conjunctival injections on two consecutive days contributed to urgent resolution of the exudate, which could have resulted in pupil block glaucoma or corneal decompensation, if left untreated. She did not respond to topical cycloplegics or steroids initially in the first half hour. Subconjunctival mydriacaine was phenomenal in partly dilating her pupil, to lead us to the correct diagnosis.

Thus, it is important in uveitis to correctly 
diagnose and treat it, to prevent significant visual morbidity that follows it. We were fortunate that at that time, the decrease in vision of our patient was attributed to her pre-existing cataracts, rather than uveitis, although it may have played a role in the causation or progression of cataracts, although the patient did not give a prior history of such episodes.

We decided to share our unique case to consider an exudate to be ectopia lentis. Extensive literature search has failed to generate a similar case of a lenslike mass in the anterior chamber.

\section{Conflict of Interest}

Authors declared no conflict of interest

\section{Authors' Designation and Contribution}

Sana Nadeem; Assistant Professor: Manuscript drafting, literature review and final review.

BA Naeem; Professor: Literature review and final review.

\section{REFERENCES}

1. Akinsoji E, Goldhardt R, Galor A. A Glimpse into Uveitis in the Aging Eye: Pathophysiology, Clinical Presentation and Treatment Considerations. Drugs Aging. 2018; 35 (5): 399-408.

Doi:10.1007/s40266-018-0545-3.
2. Dick AD, Tundia N, Sorg R, Zhao C, Chao J, Joshi A, Skup M. Risk of Ocular Complications in Patients with Noninfectious Intermediate Uveitis, Posterior Uveitis, or Panuveitis. Ophthalmology. 2016; 123 (3): 655-62.

3. Yanoff M, Duker JS. Ophthalmology. Third Edition. Mosby: St. Louis, 2009: p. 783.

4. Kheir WA, Sheheitli HA, Hamam RN. Intraocular inflammation in diabetic populations. Curr Diab Rep. 2017; 17 (10): 83.

5. Watanabe T, Keino $H$, Nakayama $K$, Taki W, Echizen N, Okada AA. Clinical features of patients with diabetic anterior uveitis. Br J Ophthalmol. 2019; 103 (1): 78-82.

Doi:10.1136/bjophthalmol-2017-311453.

6. Rothova A, Meenken C, Michels RP, Kijlstra A. Uveitis and diabetes mellitus. Am J Ophthalmol. 1988; 106 (1): 17-20.

7. Probst K, Fijnheer R, Schellkens P, Rothova A. Intraocular and plasma levels of cellular fibronectin in patients with uveitis and diabetes mellitus. $\mathrm{Br} \mathrm{J}$ Ophthalmol. 2004; 88 (5): 667-72.

8. Oswal KS, Sivaraj RR, Stavrou P, Murray PI. Clinical features of patients presenting with their first episode of uveitis. Ocul Immunol Inflamm. 2009; 17 (6): 390-3.

9. Oswal KS, Sivaraj RR, Murray PI, Stavrou P. Clinical course and visual outcome of patients with diabetes mellitus and uveitis. BMC Res Notes. 2013; 6: 167.

10. Kheir WJ, Sheheitli HA, Hamam RN. Intraocular Inflammation in Diabetic Populations. Curr Diab Rep. 2017; 17 (10): 83. 